\title{
Селективное определение водорода металлоксидным сенсором
}

\author{
Шапошник А.В., Москалев П.В, Чегерева К.Л., Звягин А.А., Сизаск Е.А. \\ Воронежский государственный аграрный университет, Воронеж
}

Поступила в редакцию 4.06.2018 г.

DOI: https://doi.org/10.17308/sorpchrom.2018.18/602

Селективное определение водорода выполнено с использованием единичного полупроводникового металлоксидного сенсора электрокондуктивного типа. Сочетание специально подобранного состава газочувствительного слоя сенсора со специально подобранным режимом температурной модуляции позволили получить зависимости электрического сопротивления сенсора от времени, имеющие характерные экстремумы. Для обработки многомерных данных использовалась специально разработанная процедура. Применение температурной модуляции позволило повысить не только селективность анализа, но также его чувствительность - отклик сенсора увеличился на одиндва порядка по сравнению с использованием стационарного температурного режима. Цель исследования - разработка метода селективного определения газов и паров с использованием малоселективного химического сенсора.

Ключевые слова: металлоксидный сенсор, водород, температурная модуляция, качественный анализ, количественный анализ.

\section{Selective determination of hydrogen by the metal oxide sensor}

\author{
Shaposhnik A.V., Moskalev P.V., Chegereva K.L., Zviagin A.A., Sizask E.A. \\ Voronezh State Agricultural University, Voronezh
}

Semiconductor sensors are commonly used to solve the important practical problem of determining the concentration of hydrogen in the air. Their disadvantage is the low selectivity, which can lead to a false alarm when the analytical device enters the atmosphere of other gases-reducing agents, for example, ethanol vapor, ammonia and so on. To increase the selectivity, the temperature modulation in combination with the selection of the gas-sensitive sensor layer was used. Due to the temperature modulation, the volume of information about each analyte was increased, because its features associated with the kinetics of sorption on the sensor surface, with the kinetics of the chemical interaction between the reducing analysts and chemisorbed oxygen, with the kinetics of the desorption of chemical interaction products were revealed. However, the information obtained in the experiment about the qualitative composition of the medium is contained in the obtained kinetic data in an implicit form, while the procedure for conducting a qualitative analysis using low-selective sensors has remained undeveloped to date. In this paper, a method of qualitative analysis was proposed, based on the determination of empirical coefficients in the equation relating the concentration of the analyte gas to the sensory response (electrical resistance) for different moments of time during the measurement cycle.

The use of temperature modulation in combination with the selection of the gas-sensitive layer composition allowed to increase the sensitivity of the quantitative analysis by one or two orders of magnitude (depending on the range of hydrogen concentrations). analysis.

Keywords: metal oxide sensor, hydrogen, temperature modulation, qualitative analysis, quantitative 


\section{Введение}

Определение водорода в воздухе является важной практической задачей, связанной с развитием водородной энергетики. Как известно, водород является наиболее перспективным топливом для городского транспорта, однако взрывоопасность его смесей с кислородом воздуха создает определённые препятствия для широкого распространения водородных двигателей. Острота этой проблемы могла бы снизиться при появлении миниатюрных и недорогих газоанализаторов нового поколения, обладающих повышенной чувствительностью и селективностью к водороду. Однако химические газовые сенсоры не обладают высокой селективностью и допускают ложное срабатывание при попадании в воздух других газов-восстановителей. Задачей данной работы явилась разработка метода качественного и количественного определения водорода с помощью химического газового сенсора.

В настоящее время применение простых в эксплуатации портативных газоанализаторов на основе химических сенсоров ограничивается их низкой селективностью. Ранее предполагалось, что задачи селективного анализа газов будут успешно решены с помощью мультисенсорных систем («электронных носов»). Однако выяснилось, что нестабильность работы подобных устройств существенно повышается при увеличении количества сенсоров. В связи с этим возникла необходимость создания устройств на основе ограниченного количества сенсоров.

Для селективного анализа газов необходимо получить достаточно большой объем информации об исследуемой газовой среде, что невозможно сделать при стационарных условиях эксперимента. Переход к нестационарным режимам увеличивает объем информации об аналите, потому что выявляет его особенности, связанные с кинетикой хемосорбции на поверхности сенсора, с кинетикой химического взаимодействия между аналитами-восстановителями и хемосорбированным кислородом, с кинетикой десорбции продуктов химического взаимодействия [1-12]. Эти отличия в неявном виде содержатся в данных по кинетике нестационарного процесса. Для проведения качественного и количественного анализа необходимо проведение хемометрической обработки массивов многомерных данных.

Цель исследования - разработка метода селективного определения газов и паров с использованием малоселективного химического сенсора.

\section{Эксперимент}

Для создания газочувствительного слоя металлоксидного сенсора использовали высокодисперсный порошок диоксида олова, полученного из оловянной кислоты. К нанодисперсному диоксиду олову добавляли нитрат тетраамминпалладия (+2). Полученный нанопорошок смешивали с вязким наполнителем, раствором метилцеллозольва в амиловом спирте, для получения пасты, которую наносили на диэлектрическую подложку, содержащую платиновые электроды и платиновый нагреватель. Подложка с нанесенным на нее тонким слоем нагревалась до температуры $750^{\circ} \mathrm{C}$, в результате чего из пасты формировался высокодисперсный хрупкий гель. Палладий, содержащийся в комплексном соединении, частично восстанавливались до металла, частично превращался в оксиды, преимущественно, в PdO. После формирования газочувствительного слоя сенсор припаивался к корпусу.

На рисунке 1 показано изменение температуры (кривая 1), изменение сопротивления сенсора при концентрации 100 ppm водорода (кривая 2) и изменение сопротивления сенсора при концентрации 100 ppm этанола (кривая 3) на протяжении 
трех циклов измерений. Длительность каждого цикла составляла 15 секунд, первые две секунды шло нагревание сенсора до $450{ }^{\circ} \mathrm{C}$, потом охлаждение до $100^{\circ} \mathrm{C}$.

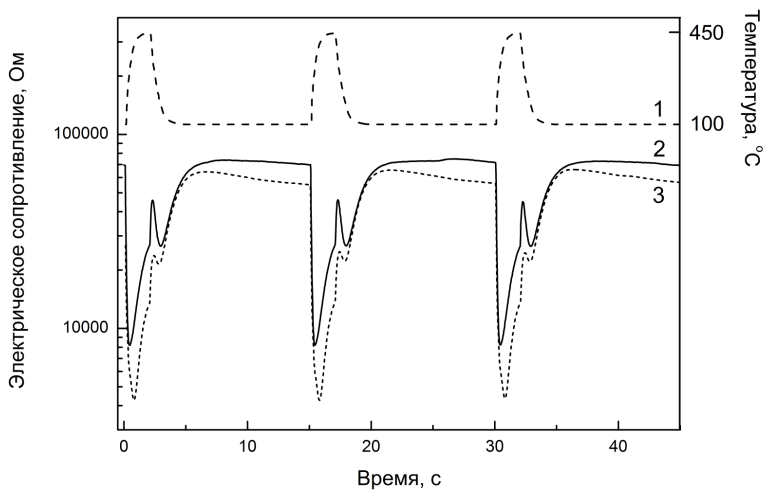

Рис. 1. Зависимость температуры и сопротивления сенсора на основе $\mathrm{SnO}_{2}$ с добавками палладия от времени на протяжении трех циклов измерений при определении водорода и этанола (пояснения в тексте)

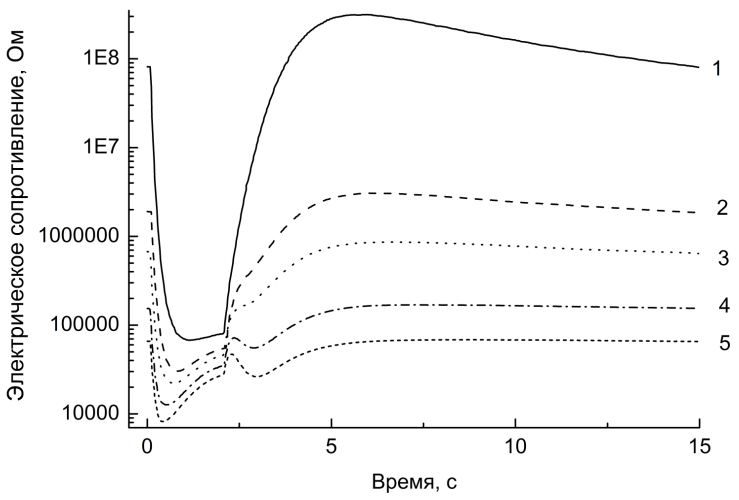

Рис. 2. Зависимость сопротивления сенсора от времени на протяжении одного цикла измерений при определении водорода различных концентраций (пояснения в тексте)

На рисунке 2 показано изменение сопротивления сенсора на протяжении одного цикла измерений для воздуха (кривая 1), 10 ppm водорода (кривая 2), 20 ppm водорода (кривая 3), 50 ppm водорода (кривая 4), 100 ppm водорода (кривая 5).

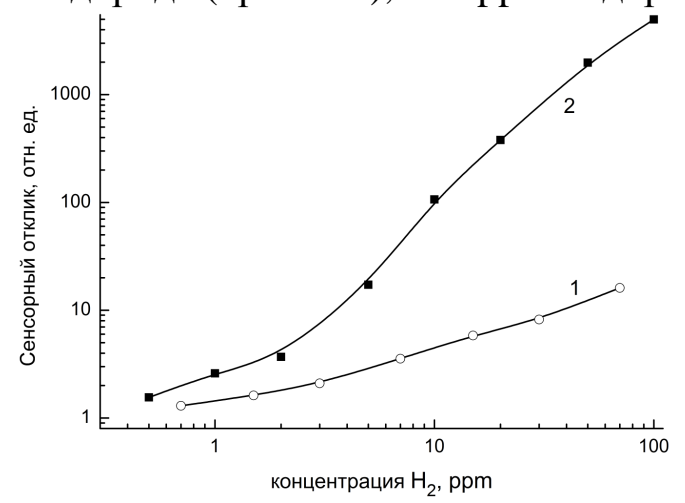

Рис. 3. Зависимость отклика сенсора от концентрации водорода в постоянном температурном режиме и в режиме температурной модуляции (пояснения в тексте).

Линия 1 на рисунке 3 показывает значения отклика сенсора, функционирующего при постоянной температуре $300^{\circ} \mathrm{C}$, линия 2 - значения отклика сенсора, функционирующего в условиях температурной модуляции. Как следует из рисунка, переход к выбранному нами импульсному температурному режиму приводит к увеличению откликов на один-два порядка в диапазоне концентраций от 10 до 100 ppm и к соответствующему повышению чувствительности. Для металлоксидных сенсоров хороший отклик к газам-восстановителям примерно соответствует концентрации газа, выраженной в ppm. Таким образом, высокий отклик к 100 pрm водорода должен достигать величины 100 относительных единиц, однако в нашем случае он больше в пятьдесят раз.

Решение задачи проведения качественного анализа было разбито на два этапа. Сначала методом наименьших квадратов были определены эмпирические коэффициенты $a$ и $b$ в уравнении, связывающем концентрацию газа-аналита $\varphi$ со 
значениями сопротивления сенсора $R$ в различные моменты времени на протяжении цикла измерений (Рис. 2):

$$
\varphi=a \cdot R^{b}
$$

Всего из цикла продолжительностью 15 секунд были взяты 60 значений сопротивления $R$ и получены 60 пар значений эмпирических коэффициентов $a$ и $b$, при этом использовались результаты, вошедшие в обучающую выборку.

В дальнейшем для проверки были использованы тестовые результаты, не вошедшие в обучающую выборку. Для каждого тестового анализа было взято 60 значений сопротивления, соответствующие различным моментам времени на протяжении цикла измерений. На основе найденных при анализе значений сопротивления были получены 60 значений концентрации $\varphi$ при решении уравнения (1). Из этих 60 значений было определено среднее (количественный анализ) и относительное стандартное отклонение. Процедура проведения качественного анализа заключалась в сравнении величин относительного стандартного отклонения (табл.). Если относительное стандартное отклонение имело значение менее 0.5 , то предполагалось, что газом-аналитом является водород, если более 0.5 - какой-то другой газ.

Таблица. Относительные стандартные отклонения определения концентрации водорода для тестовых анализов.

\begin{tabular}{|c|c|c|}
\hline $\begin{array}{l}\text { Тестовые измерения } \\
\text { концентрации }\end{array}$ & $\begin{array}{c}\text { Полученные значения } \\
\text { концентрации, ppm }\end{array}$ & $\begin{array}{c}\text { Относительные стандартные } \\
\text { отклонения }\end{array}$ \\
\hline $15.6 \mathrm{ppm} \mathrm{H}_{2}$ & 14.5 & 0.168 \\
\hline $29.0 \mathrm{ppm} \mathrm{H}_{2}$ & 27.4 & 0.051 \\
\hline $77.4 \mathrm{ppm} \mathrm{H}_{2}$ & 77.7 & 0.026 \\
\hline $14.0 \mathrm{ppm} \mathrm{C}_{2} \mathrm{H}_{5} \mathrm{OH}$ & - & 0.713 \\
\hline $60.0 \mathrm{ppm} \mathrm{C}_{2} \mathrm{H}_{5} \mathrm{OH}$ & - & 1.016 \\
\hline $80.0 \mathrm{ppm} \mathrm{C}_{2} \mathrm{H}_{5} \mathrm{OH}$ & - & 2.167 \\
\hline
\end{tabular}

Как показано в третьей колонке таблицы 1, для всех тестовых анализов водорода величина относительного стандартного отклонения принимала значения менее 0.5, а для всех тестовых анализов этанола принимала значения более 0.5 . Таким образом, нам удалось провести качественный анализ водорода, не допустив ложного определения в тех случаях, когда вместо водорода в исследовательскую камеру поступали пары этанола. Полученные нами значения концентрации водорода (вторая колонка таблицы 1) близко совпали с истинными значениями (первая колонка таблицы).

\section{Обсуждение результатов}

В чем причина полученных в работе рекордных значений чувствительности? Снижение температуры газочувствительного слоя приводит к увеличению сорбции анализируемого газа, однако при низких температурах молекулы не переходят в активное состояние, необходимое для протекания химических процессов. При высокой температуре молекулы водорода переходят в активное состояние и способны взаимодействовать с находящимися на поверхности газочувствительного слоя анионами кислорода $O_{2}^{-}, O^{-}$или $\mathrm{O}^{2-}$ :

$$
\mathrm{H}_{2}+\mathrm{O}^{-} \rightarrow \mathrm{H}_{2} \mathrm{O}+\bar{e}
$$


Однако повышение температуры снижает величину сорбции анализируемого газа. Таким образом, определение сенсорного отклика в стационарном режиме требует поиска компромисса - повышение температуры снижает сорбцию, а снижение температуры пассивирует катализатор и уменьшает количество активных молекул.

Использование выбранного нами импульсного режима позволяет совместить преимущества разных температур газочувствительного слоя. В начале цикла сенсор охлажден и величина сорбции водорода максимальна. Нагрев сенсора до достаточно высокой температуры $450^{\circ} \mathrm{C}$ позволяет активировать катализатор, перевести большое количество молекул водорода в активное состояние и заставить их взаимодействовать с избытком анионов кислорода (2). Резкий нагрев газочувствительного слоя приводит к тому, что молекулы водорода успевают активироваться и вступить во взаимодействие с анионами кислорода раньше, чем произойдет их десорбция с поверхности. Именно этим объясняется аномальное увеличение отклика при использовании резкого, ступенчатого нагрева. Подобное увеличение отклика не наблюдалось при использовании плавного синусоидального температурного режима.

\section{Заключение}

Приведенные результаты показывают принципиальную возможность проведения качественного и количественного анализа на основе единичного малоселективного сенсора. Это может быть достигнуть за счет использования температурной модуляции в сочетании с подбором состава газочувсвительного слоя и последующей обработкой многомерных данных. Чувствительность сенсора в выбранном режиме возрастает на один-два порядка по сравнению с постоянным температурным режимом.

\section{Список литературы/References}

1. Shaposhnik A.V, Zviagin A.A., Sizask E.A. et al., Procedia Engineering, 2014, Vol. 87, pp. 1051-1054. doi: 10.1016/j.proeng.2014.11.343.

2. Samotaev N.N., Vasiliev A.A., Podlepetsky B.I., Sokolov A.V. et al, Sensors and Actuators $B$, 2007, Vol. 127, pp. 242-247. doi:10.1016/j.snb.2007.07.022.

3. Weimar U., Schierbaum K.D., Göpel W., Kowalkowski R., Sensors and Actuators B, 1990, Vol. 1, No 1-6, pp. 93-96.

4. Heilig A., Barsan N., Weimar U., Schweizer-Berberich M. et al., Sensors and Actuators B, 1997, Vol. 43, pp. 45-51.

5. Nakata S., Ozaki E., Ojima N., Analytica Chimica Acta, 1998, Vol. 361, pp. 93-100.

6. Nakata S., Nakasuji M., Ojima N., Kitora M., Applied Surface Science, 1998, Vol. 135, pp. 285-292.

Шапошник Алексей Владимирович - д.х.н., заведующий кафедрой химии Воронежский аграрный университет им. императора Петра I, Воронеж.
7. Nakata S., Takemuri K., Neya K., Analytical sciences (The Japan Society for Analytical Chemistry), 2001, Vol. 17, pp. 365-373.

8. Nakata S., Neya K., Takemura K.K., Thin Solid Films, 2001, Vol. 391, pp. 293-298.

9. Radu Ionescu, Eduard Llobet, Sensors and Actuators B, 2002, Vol. 81, pp. 289-295.

10. Ionescu R., Llobet E., Brezmes J., Vilanova X. et al., Sensors and Actuators B, 2003, Vol. 95, pp. 177-182. doi:10.1016/S09254005(03)00411-8.

11. Hui Ding, Haifeng Ge, Junhua Liu, Sensors and Actuators B, 2005, Vol. 107, pp. 749755. doi:10.1016/j.snb.2004.12.009.

12. Haifeng Ge, Junhua Liu, Sensors and Actuators $B, 2006$, Vol. 117, pp. 408-414. doi:10.1016/j.snb.2005.11.037.

\footnotetext{
Shaposhnik Alexey A. - head of chair of chemistry, Voronezh State Agrarian University, Voronezh.
} 
Чегерева Кристина Леонидовна - аспирант кафедры химии Воронежский аграрный университет им.императора Петра I, Воронеж

Москалев Павел Валентинович - д.ф.-м.н. профессор кафедры математики и физики, Воронежский аграрный университет им. императора Петра I, Воронеж.

Звягин Алексей Алексеевич - доцент кафедры химии, Воронежский аграрный университет им.императора Петра I, Воронеж

Сизаск Елена Александровна - аспирант кафедры химии Воронежский аграрный университет им.императора Петра I, Воронеж
Chegereva Kristina L. - postgraduate student, chair of chemistry, Voronezh State Agrarian University. Voronezh.

Moskalev Pavel V. - prof., grand Ph.D. (physics and mathematics), department of mathematics and physics, Voronezh State Agrarian University, Voronezh; e-mail: moskalefff@gmail.com

Zviagin Alexey A. - docent, chair of chemistry, Voronezh State Agrarian University, Voronezh.

Sizask Elena A. - postgraduate student, chair of chemistry, Voronezh State Agrarian University. Voronezh. 